\title{
Estimating pKa shifts of encapsulated drugs through a CpHMD approach
}

Diogo Reis, Miguel Machuqueiro and Diogo Vila-Viçosa

Centro de Química e Bioquímica and BioISI: Biosystems and Integrative Sciences Institute,

Faculdade de Ciências, Universidade de Lisboa, 1749-016 Lisboa, Portugal

Email: diogoreis992@gmail.com

Molecular machines have recently been associated with the development of molecular carriers to enhance drug properties, such as solubility or bioavailability. One possible approach is through drug encapsulation by a host molecule, such as cucurbituril (CB) rings, which modifies the environment of the guest molecule. CB rings are able to encapsulate guest molecules providing a hydrophobic cavity and several carbonyl groups that stabilize cationic hosts that interact with this region. This results in significant $p K_{a}$ shifts for drugs with titrable (cationic) groups that can be exploited in order to improve drug bioavailability, whether by enhancing their solubility, stabilizing their active form or by protecting them against external agents. The aforementioned approach can be used for medical targeting, such as cancer therapy, by designing carriers that deliver guest molecules at specific conditions, knowing the specific target properties [1].

Computational tools are a powerful way to help the rational design of CB-guest complexes. In particular, the stochastic titration constant-pH MD (CpHMD) method allows a molecular dynamics simulation to have the $\mathrm{pH}$ value as an external parameter and, consequently, obtain full titration curves and $\mathrm{p} K_{\mathrm{a}}$ values. Our main goal is to develop a strategy to model benzimidazole (BZ) $p K_{a}$ shifts, a «proof-of-concept» molecule, and then extrapolate this process to other host-guest complexes. BZ has a well-known shift of $\sim 3.5 \mathrm{pK} K_{\mathrm{a}}$ units when encapsulated by a CB ring and, with a CpHMD method, it is possible to elucidate the molecular details of these host-guest interactions.

\section{Acknowledgments}

We acknowledge financial support from FCT through project UID/MULTI/00612/2013 and grant SFRH/BPD/110491/2015.

\section{References}

1. I. Ghosh, W. M. Nau, "The strategic use of supramolecular $p K_{a}$ shifts to enhance the bioavailability of drugs." Advanced Drug Delivery Reviews, vol. 64, pp. 764-783, 2012. 